\title{
The condition for the maximum of the generalized power function in the problem of forming a multi-mode control law with limitation
}

\author{
Andrey Kostoglotov $^{1,2,3}$, Sergey Lazarenko ${ }^{1,2,3^{*}}$, Alexander Agapov ${ }^{l}$ and Zoya Lyashchenko $^{2}$ \\ ${ }^{1}$ Don State Technical University, 344003, Gagarin sq., 1, Rostov-on-Don, Russia \\ ${ }^{2}$ Rostov State Transport University, 344038, Rostovskogo Strelkovogo Polka Narodnogo \\ Opolcheniya sq., 2, Rostov-on-Don, Russia \\ ${ }^{3}$ Moscow State University of Technology and Management named after K.G. Razumovsky, 109004, \\ Zemlyanoy Val st., 73, Moscow, Russia
}

\begin{abstract}
A quasi-optimal control law is developed based on the condition for the maxi-mum of the generalized power function taking into account the stationarity of the Hamiltonian on the switching line for control objects that can be represented by the Lagrange equations of the second kind. The comparative analysis is carried out based on the mathematical simulation using the optimal nonlinear control laws with respect to several criteria. We found that the modes of the proposed control law provide high accuracy of approximation to the optimal performance laws and the Fuller laws, reducing energy costs for control by eliminating more frequent switching. The choice of the parameters of the developed control law makes it possible to implement a wide range of both nonlinear and linear operating modes, which allows to classify the obtained control law as multimode law.
\end{abstract}

\section{Introduction}

The synthesis of control laws that are used in the construction of multi-mode systems can be based on the use of the Lyapunov methods, the methods of optimal synthesis using the principles of L. Pontryagin and R. Bellman, the empirical solutions using and generalizing the control experience that require methods of intellectualization [1-5]. The latter are not effective enough in the case when it is possible to build an accurate mathematical model [6-8].

We can assert that the set of solutions that ensure the stability of the synthesized system is infinite, while there are significantly fewer optimal solutions to the synthesis problem satisfying a given criterion. Their set is limited, therefore, it is not always possible to use them to construct multi-mode systems. So it is necessary to search for quasi-optimal control laws that are close to optimal laws with respect to a given criterion [9].

Works [10-14] consider an approach that allows to obtain quasi-optimal solutions to the synthesis problem based on the condition for the maximum of the generalized power

\footnotetext{
* Corresponding author: lazarenkosv@icloud.com
} 
function for a wide class of dynamical systems that satisfy the Hamilton-Ostrogradskii principle and, accordingly, can be represented by the Lagrange equations of the second kind.

The aim of this work is to construct a multi-mode control based on the proposed approach and to compare the obtained solutions with classical optimal solutions.

\section{Formulation of the control problem}

Let us consider a class of controlled systems which motion in independent coordinates $q=\left\|q_{s}\right\|_{s=1}^{n}$ can be described by the Lagrange differential equations of the second kind

$$
\frac{d}{d t} \frac{\partial T}{\partial \dot{q}_{s}}-\frac{\partial T}{\partial q_{s}}=u_{s}(t), \quad s=\overline{1, n} .
$$

The kinetic energy $T$ of each system of this class is selected from the set of positive definite quadratic forms of generalized velocities $\dot{q}_{s}$

$$
\begin{aligned}
& T=\frac{1}{2} \sum_{s, k=1}^{n} a_{s k}(q) \dot{q}_{s} \dot{q}_{k}, \quad \eta_{0} \sum_{s=1}^{n} \dot{q}_{s}^{2} \leq T \leq \eta_{1} \sum_{s=1}^{n} \dot{q}_{s}^{2} \\
& \eta_{0,1}=\text { const }, \quad \eta_{0,1}>0
\end{aligned}
$$

with continuously differentiable coefficients $a_{s k}(q)$. In this case, the coefficients $a_{s k}(q)$ can also depend on constant parameters (masses, moments of inertia, geometric dimensions, etc.). Since arbitrary functions are admitted to consideration $a_{s k}(q)$, for which the inequality (2) is valid, we will not consider the dependence of the coefficients $a_{s k}$ on constant parameters.

The admissible controls are selected from the set of functions summable on any finite interval of functions $u(t)=\left\|u_{s}(t)\right\|_{s=1}^{n}, \quad t$ is the current time, taking values in a bounded closed convex region $U, u(t) \in U, \operatorname{Conv} U=U$, where $\operatorname{Conv} U$ is the convex closure of the set $U$.

For simplicity we will assume that the convex region $U$. of variation of admissible controls is a parallelepiped, i.e.

$$
u(t) \in U=\left\{u_{s}(t),\left|u_{s}(t)\right| \leq h_{s}, s=\overline{1, n}\right\} .
$$

The specified class of controllable Lagrangian systems is given by the set $U$ and by the constant parameters $\eta_{0}$ and $\eta_{1}$. The specific system of this class is distinguished by the definition of the quadratic form $T$ in accordance with (2). Any such system is considered to belong to this class.

We assume that the system can be affected by any forces included in the set of the limited controls $u(t) \in U$, i.e. the system is fully controllable [15]: $\min _{1 \leq s \leq n} h_{s}>0$. 
The optimal control problem is to transfer system (1), (2) from the initial state

to the final state

$$
t=t_{0}, \mathbf{q}\left(t_{0}\right)=\left[q_{10}, \ldots, q_{n 0}\right]^{T}, \dot{\mathbf{q}}\left(t_{0}\right)=\left[\dot{q}_{10}, \ldots, \dot{q}_{n 0}\right]^{T}
$$

$$
t=t_{k}, \mathbf{q}\left(t_{k}\right)=\left[q_{1 k}, \ldots, q_{n k}\right]^{T}, \dot{\mathbf{q}}\left(t_{k}\right)=\left[\dot{q}_{1 k}, \ldots, \dot{q}_{n k}\right]^{T}
$$

subject to the minimum of the target functional

$$
J(\mathbf{q})=\int_{t_{0}}^{t_{1}} F(\mathbf{q}) d t \rightarrow \min ,
$$

where $\quad F(\mathbf{q})$ is a positive definite convex function of generalized coordinates.

This paper formulates the problem to construct a multi-mode control for the system (1), (2) subject to condition (3), (6) on the set of the quasi-optimal control laws obtained from the condition for the maximum of the generalized power function [10-15].

\section{Constructing the multi-mode control law}

A quasi-optimal control law, constructed using the condition for the maximum function of the generalized power, can be represented in the form [15]:

$$
\frac{d}{d t} \frac{\partial T}{\partial \dot{q}_{s}}-\frac{\partial T}{\partial q_{s}}=u_{s}\left(q_{s}, \dot{q}_{s}\right)=s a t\left\{\lambda^{-1}\left[\mu_{s}(q, \dot{q}) \dot{q}_{s}+\frac{\partial F}{\partial q_{s}}\right]\right\},
$$

where $\mu_{s}(q, \dot{q})$ is the synthesizing function, one of the requirements for which is having definite sign, taking into account the limitation of the control signal (3)

$$
\operatorname{sat}\left(u_{s}\left(q_{s}, \dot{q}_{s}\right)\right)= \begin{cases}u_{s}\left(q_{s}, \dot{q}_{s}\right), & \left|u_{s}\left(q_{s}, \dot{q}_{s}\right)\right| \leq h_{s}, \\ h_{s} \operatorname{sgn}\left(u_{s}\left(q_{s}, \dot{q}_{s}\right)\right), & \left|u_{s}\left(q_{s}, \dot{q}_{s}\right)\right|>h_{s} .\end{cases}
$$

Method of constructing the synthesizing function can be based on the assumption that one of the first integrals of motion of the system is stationary. For example, we can take the generalized Hamilton function

$$
H(q, p)=\lambda(T+A)+F=\text { const. }
$$

We should note that in the absence of control forces $A=0$ the system under consideration can be classified as autonomous conservative system, since $F(\mathbf{q})$ is the positive definite convex function of the generalized coordinates.

The work $A$ depends on the control forces (7). Now we consider the conditions that with $A=0$ determine the switching line 


$$
\begin{gathered}
u_{0}=\lambda^{-1}\left[\mu_{s}(q, \dot{q}) \dot{q}_{s}+\frac{\partial F}{\partial q_{s}}\right]=0, \\
H_{0}=\lambda T+F=\mathrm{const}
\end{gathered}
$$

and, accordingly, the type of synthesizing function.

The function $\mu_{s}(q, \dot{q}) \dot{q}_{s}+\frac{\partial F}{\partial q_{s}}$ is a function of coordinates $q_{s}$ and momenta $p_{s}$. If we require that the Poisson brackets for the quantities $u_{0}$ and $H_{0}$ are

$$
\left[u_{0}, H_{0}\right]=\frac{\partial H_{0}}{\partial q_{s}} \cdot \frac{\partial u_{0}}{\partial p_{s}}-\frac{\partial H_{0}}{\partial p_{s}} \cdot \frac{\partial u_{0}}{\partial q_{s}}=0
$$

then the curve

$$
\mu_{s} \dot{q}_{s}+\frac{\partial F}{\partial q_{s}}=0
$$

in the phase space determines the integral of motion, which is explicitly independent of time, and allows to obtain the expression for the synthesizing function. Thus, the switching curve and the synthesizing function are determined by the integral of motion, provided that work of the external forces vanishes.

For the case of the main generalized coordinates and the constant coefficients of inertia, from (16) we obtain:

$$
\frac{\partial F}{\partial q_{s}} \mu_{s}-\lambda \dot{q} \frac{\partial^{2} F}{\partial q_{s}^{2}}=0, \quad \mu_{s}=\lambda \dot{q}_{s} \frac{\partial^{2} F}{\partial q_{s}^{2}} / \frac{\partial F}{\partial q_{s}}
$$

Then, taking into account the expression (17) and the condition of definite sign of the synthesizing function [15], we have on the switching line

$$
\sqrt{\lambda\left(\frac{\partial^{2} F}{\partial q_{s}^{2}}\right)} \dot{q}_{s}= \pm\left(\frac{\partial F}{\partial q_{s}}\right)
$$

Therefore, on the switching line the velocities are limited by the kinematic constraints.

When implementing the multi-mode control, to ensure the holding kinematic constraints it is required, according to the release principle, to apply the reaction forces of the constraints [16]

$$
R_{s}=\varepsilon_{s} l_{s}
$$

where $\varepsilon_{s}$ are called the Lagrange multipliers of the second kind or constraint factors, $l_{s}=\sqrt{\lambda\left(\frac{\partial^{2} F}{\partial q_{s}^{2}}\right)}$ is a coefficient that is determined by the type of kinematic constraint (14). 
The elementary work of these forces on virtual displacements has the form

$$
\delta^{\prime} E=\sum_{s=1}^{n} R_{s} \delta q_{s} .
$$

These forces are taken into account by including their work in the action integral, so the extended functional takes the form

$$
J=\int_{t_{0}}^{t_{1}}[\lambda T+F+E] d t=\int_{t_{0}}^{t_{1}} H^{\prime}(q, p) d t .
$$

Then, for the switching line, we have taking into account the Poisson brackets and definite sign of the synthesizing function,

$$
\begin{aligned}
& {\left[\frac{\partial F_{s}}{\partial q_{s}}+\varepsilon_{s} \sqrt{\lambda\left(\frac{\partial^{2} F}{\partial q_{s}^{2}}\right)}\right] \mu_{s}-\lambda \dot{q}_{s} \frac{\partial^{2} F}{\partial q_{s}^{2}}=0,} \\
& \mu_{s}=-\lambda\left|\dot{q}_{s}\right|\left[\left|\frac{\partial^{2} F}{\partial q_{s}^{2}}\right| / \mid \frac{\partial F}{\partial q_{s}}+\varepsilon_{s} \sqrt{\left.\lambda\left(\frac{\partial^{2} F}{\partial q_{s}^{2}}\right) \mid\right] .}\right.
\end{aligned}
$$

\section{Mathematical simulation}

Let us consider the object control problem

with limited control

$$
\ddot{x}=u
$$

$$
|u| \leq h, h=1
$$

and given initial conditions

$$
\left[\begin{array}{c}
x_{0} \\
\dot{x}_{0}
\end{array}\right]=\left[\begin{array}{c}
x(0) \\
\dot{x}(0)
\end{array}\right] .
$$

The optimal solution for the performance problem

$$
J(u(x, \dot{x}))=\int_{t_{0}}^{t_{1}} d t \rightarrow \inf
$$

is the Pontryagin control law

$$
u_{\text {pon }}(x, \dot{x})=h \operatorname{sign}\left[x+\frac{\dot{x}|\dot{x}|}{2 h}\right] .
$$

Optimal solution with respect to the squared deviation criterion (the Fuller problem) 


$$
J(u(x, \dot{x}))=\int_{t_{0}}^{t_{1}} x^{2} d t \rightarrow \inf
$$

is a solution in the form of a control with increasing switching

$$
u_{\text {full }}(x, \dot{x})=h \operatorname{sign}\left[x+0.4446 \frac{\dot{x}|\dot{x}|}{h}\right] \text {. }
$$

The control law obtained on the basis of the condition for the maximum of the generalized power Ошибка! Источник ссылки не найден. has the form

$$
u_{c m p}(x, \dot{x})=s a t\left[k_{1}\left(x+k_{2} \frac{\dot{x}|\dot{x}|}{|x|+k_{3}}\right)\right]
$$

where $k_{1}, k_{2}, k_{3}$ are the parameters of the control law;

$$
\operatorname{sat}(f)= \begin{cases}f, & |f| \leq h \\ h \operatorname{sgn}(f), & |f|>h .\end{cases}
$$

Within the framework of numerical simulation the several modes of the controller operation are considered (26). The estimation of the proximity of quasi-optimal and optimal control processes is carried out for the initial conditions $\left[\begin{array}{c}x_{0} \\ \dot{x}_{0}\end{array}\right]=\left[\begin{array}{c}-0,2 \\ 0,2\end{array}\right]$ and the sampling interval $\Delta t=0,001$ based on the following indicators.

Relative deviation of the performance functional

$$
\delta J_{\text {time }}=\frac{J_{\text {time }}\left(u_{\text {cmp }}\right)-J_{\text {time }}\left(u_{\text {aim }}\right)}{J_{\text {time }}\left(u_{\text {aim }}\right)} \cdot 100 \%,
$$

where $J_{\text {time }}$ is the value of the performance functional (22), $u_{c m p}$ is the proposed control law, $u_{\text {aim }}$ is the optimal control law.

Relative deviation of the quadratic functional

$$
\delta J_{x}=\frac{J_{x}\left(u_{c m p}\right)-J_{x}\left(u_{\text {aim }}\right)}{J_{x}\left(u_{\text {aim }}\right)} \cdot 100 \%,
$$

where $J_{x}$ is the value of the quadratic functional (24).

The relative change in energy costs is determined in accordance with the expression 


$$
\delta J_{u}=\frac{J_{u}\left(u_{c m p}\right)-J_{u}\left(u_{\text {aim }}\right)}{J_{u}\left(u_{\text {aim }}\right)} \cdot 100 \%,
$$

where $J_{u}$ is the value of the functional

$$
J(u(x, \dot{x}))=\int_{t_{0}}^{t_{1}} u^{2} d t
$$

Let us consider various modes of the system (31) controlled by the proposed law (38).

The choice of the parameters $k_{1}=1000, k_{2}=0,56, k_{3}=1$ implements a mode which is quasi-optimal mode with respect to the performance criterion when it is close to optimal mode with an accuracy $\delta J_{\text {time }}=1,92 \%$.

The choice of the parameters $k_{1}=1060, k_{2}=0,53, k_{3}=1$ implements a mode which is quasi-optimal mode with respect to the quadratic criterion with an accuracy $\delta J_{x}=0,255 \%$.

The choice of the parameters of the obtained control law allows to realize a wide range of modes, for example, the parameters $k_{1}=13, k_{2}=0,07, k_{3}=0,0001$ ensure system behavior with precision $\delta J_{\text {time }}=1,372 \%, \delta J_{u}=-2.941 \%$ in comparison with mode which is optimal mode with respect to performance. The behavior of the system on the phase plane and the control signal are shown in Figure 1.

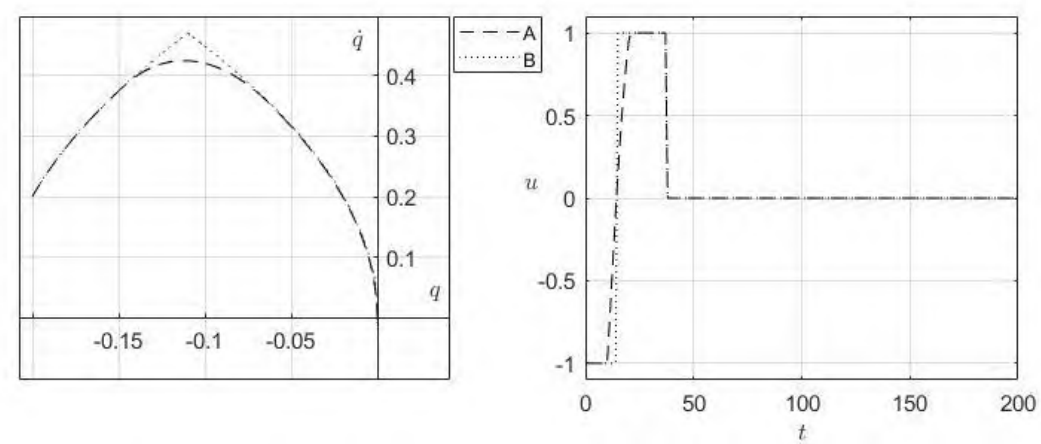

Fig. 1. Phase plane. The developed control law is mode $1(\mathrm{~A})$, optimal relay control in terms of performance (B)

The obtained control law makes it possible to provide quasi-optimal nonlinear control modes close to optimal modes in terms of various efficiency criteria, for example, the performance laws and the Fuller laws. In addition, the proposed law not only ensures proximity to the optimal law, but also delivers some gain in energy costs for control. Moreover, on the basis of the proposed structure, a wide range of linear controllers can be implemented by choosing the parameters of the law.

\section{Conclusions}


The structure of the control law, synthesized based on the condition for the maximum of the generalized power function, allows the implementation of linear and nonlinear operating modes of the controller by changing the shape of the switching line in accordance with the selected quality criterion. So we can get results close to optimal with respect to various efficiency criteria while reducing control costs.

Further research is related to the need to take into account random actions and to analyze combinations of various control modes based on the condition for the maximum of the function of generalized power in accordance with the principles of fuzzy control.

Acknowledgments: The reported study was funded by RFBR, projects number 19-3190134, 18-01-00385 A, 18-08-01494 A.

\section{References}

1. S. N. Vasiliev, Yu. I. Kudinov, F. F. Pashchenko, I. S. Durgaryan, A. Yu. Kelina, I. Yu. Kudinov, et al., Intelligent control systems and fuzzy regulators, Part II. Learning fuzzy regulators fuzzy PID regulators Sensors and systems, 3(211), 3-12 (2017)

2. B. Wang, J. Xu, B. Cao, Q. Li, Q. Yang, Adaptive Mode Control Strategy of a Multimode Hybrid Energy Storage System. Energy Procedia, 88, 627-633 (2016) ISSN 1876-6102, https://doi.org/10.1016/j.egypro.2016.06.089.

3. H. I. Jaafar, Z. Mohamed, M. A. Shamsudin, N. A. Mohd Subha, L. Ramli, A. M. Abdullahi, Model reference command shaping for vibration control of multimode flexible systems with application to a double-pendulum overhead crane. Mechanical Systems and Signal Processing, 115, 677-695 (2019) ISSN 0888-3270, https://doi.org/10.1016/j.ymssp.2018.06.005.

4. H. Wu, J. Zhao, Self-adaptive deep learning for multimode process monitoring, Computers \& Chemical Engineering, 141, 107024 (2020) ISSN 0098-1354, https://doi.org/10.1016/j.compchemeng.2020.107024.

5. S. Nüesch, A. G. Stefanopoulou, Multimode combustion in a mild hybrid electric vehicle. Part 1: Supervisory control, Control Engineering Practice, 57, 99-110 (2016) ISSN 0967-0661, https://doi.org/10.1016/j.conengprac.2016.09.002.

6. T. Hausberger, A. Kugi, A. Eder, W. Kemmetmüller, High-speed nonlinear model predictive control of an interleaved switching DC/DC-converter, Control Engineering Practice, 103, $104576 \quad$ (2020) ISSN 0967-0661, https://doi.org/10.1016/j.conengprac.2020.104576.

7. J. B. Rawlings, D. Q. Mayne, M. Diehl, Model Predictive Control: Theory, Computation, and Design (Nob Hill Publishing, 2017)

8. S. Vazquez, J. Rodriguez, M. Rivera, L. G. Franquelo, M. Norambuena, "Model Predictive Control for Power Converters and Drives: Advances and Trends," in IEEE Transactions on Industrial Electronics, 64(2), 935-947 (2017) doi: 10.1109/TIE.2016.2625238.

9. W. Kemmetmüller, S. Eberharter, A. Kugi, Quasi optimal feedforward control of a very low frequency high-voltage test system, IFAC Proceedings Volumes, 47(3), 11623-11628 (2014) ISSN 1474-6670, ISBN 9783902823625, https://doi.org/10.3182/20140824-6-ZA-1003.00356. 
10. A. A. Kostoglotov, S. V. Lazarenko, I. V. Deryabkin, Combined maximum principle as the part of intellectualization of control systems for a suspension of vehicles. Advances in Intelligent Systems and Computing, 679, 150 (2017)

11. A. Kostoglotov, S. Lazarenko, I. Deryabkin, O. Manaenkova, A. Kuzin, I. Pugachev, Fuzzy control laws in the basis of solutions of synthesis problems of the combined maximum principle. Advances in Intelligent Systems and Computing 679, 322-329 (2018)

12. A. Kostoglotov, S. Lazarenko, I. Deryabkin, A. Yachmenov, O. Kuznetcova, Combined maximum principle as the basis of intellectualization of control systems for a suspension of vehicles. Advances in Intelligent Systems and Computing, 679, 150155 (2018)

13. A. Kostoglotov, V. Taran, V. Trofimenko, Fuzzy topological approach to a solid control task. Advances in Intelligent Systems and Computing, 874, 373-381 (2019)

14. A. Kostoglotov, S. Lazarenko, A. Agapov, Z. Lyaschenko, I. Pavlova, Designing the knowledge base for the intelligent inertial regulator based on quasi-optimal synthesis of controls using the combined maximum principle. Advances in Intelligent Systems and Computing, 874, 190-200 (2019)

15. I. Anan'evskii, S. Reshmin, Decomposition-based continuous control of mechanical systems. Journal of Computer and Systems Sciences International, 53, 473-486. (2014) 10.1134/S1064230714040029.

16. W. Nakpim, S. V. Meleshko, Conservation laws of the one-dimensional equations of relativistic gas dynamics in Lagrangian coordinates. International Journal of NonLinear Mechanics, 124, 103496 https://doi.org/10.1016/j.ijnonlinmec.2020.103496. 